\title{
BOUNDARY CONTROL SYSTEMS AND THE SYSTEM NODE
}

\author{
J.A. Villegas*, Y. Le Gorrec ${ }^{* *}$, H. Zwart*, and
}

A.J. van der Schaft*

\author{
* University of Twente, Department of Applied \\ Mathematics, PO Box 217, 7500 AE, Enschede, The \\ Netherlands. \\ $\{j . a . v i l l e g a s, h . j . z w a r t, a . j . v a n d e r s c h a f t\} @ m a t h . u t w e n t e . n l$ \\ ** LAGEP, UCB Lyon 1 - UMR CNRS 500\%, Bât. 308 G \\ 43, Bd du 11 nov. 1918, 69622 Villeurbanne cedex France, \\ legorrec@lagep.cpe.fr
}

\begin{abstract}
In this paper we show how to formulate a boundary control system in terms of the system node, that is, as an operator $\mathcal{S}:=\left[\begin{array}{c}A \& B \\ C \& D\end{array}\right]: D(S) \rightarrow\left[\begin{array}{l}X \\ Y\end{array}\right]$ where $X$ is the state space and $Y$ is the output space. Here we give results which show how to find the top part of this operator and its domain in an easy way. For a class of boundary control systems, associated with a skew-symmetric differential operator, we completely identify the system node. Some results about stability and approximate observability are presented for this class of systems. Copyright (c) 2005 IFAC
\end{abstract}

Keywords: Boundary value problem, distributed-parameter systems, controllability, stability.

\section{INTRODUCTION}

Many finite- and infinite-dimensional linear systems can be described by the equations

$$
\begin{aligned}
\dot{x}(t) & =A x(t)+B u(t) \\
y(t) & =C x(t)+D u(t), \quad t \geq 0, \\
x(0) & =x_{0}
\end{aligned}
$$

on the Hilbert spaces, namely, the input space $U$, the state space $X$ and the output space $Y$, where $u(t) \in U, x(t) \in X$ and $y(t) \in Y$. The operator $A$ is generally the generator of a $\mathrm{C}_{0}$-semigroup. Here the operators $B$ and $C$ are not necessarily bounded.

The system node (see (Staffans, 2005), (Malinen et al., 2003, §2)) has been introduced as a generalization of this set of equations for the infinitedimensional case. The system node can be thought of as the block operator $\mathcal{S}=\left[\begin{array}{c}A \& B \\ C \& D\end{array}\right]$ from $X \times U$ to $X \times Y$, which allows to replace equation (1) by

$$
\left(\begin{array}{l}
\dot{x}(t) \\
y(t)
\end{array}\right)=\mathcal{S}\left(\begin{array}{l}
x(t) \\
u(t)
\end{array}\right), \quad t \geq 0, \quad x(0)=x_{0} .
$$

However, it is not obvious how to represent boundary control systems of the form (Curtain and Zwart, 1995, §3.3)

$$
\begin{aligned}
\dot{x}(t) & =\mathfrak{A} x(t) \\
u(t) & =\mathfrak{B} x(t) \\
y(t) & =C x(t),
\end{aligned}
$$

either as the set of equations (1) or as a system node.

This paper describes how to formulate a boundary control system (BCS) as a system node. It presents results that are easy to apply. Finally, we 
focus on BCS associated with a skew-symmetric differential operator.

The organization of the paper is as follows. In Section 2 the definition of system node is given, as well as some of its properties. In Section 3 our class of boundary control systems will be presented. The relation between system nodes and BCS is presented in Section 4, together with the results that help to formulate BCS as a system node. Here we use the notation $\left[\begin{array}{c}X \\ Y\end{array}\right]$ for $X \times Y$ and $F_{\mid \mathfrak{D}}$ denotes the restriction of an operator $F$ to the subspace $\mathfrak{D} . \rho(F)$ denotes the resolvent set of $F . H^{N}\left((a, b) ; \mathbb{R}^{n}\right)$ denotes the space of $N$-times differentiable $L_{2}\left((a, b) ; \mathbb{R}^{n}\right)$ functions. $\mathbb{R}^{+}$and $\mathbb{R}^{-}$are the set of nonzero positive and negative numbers, respectively.

\section{SYSTEM NODE}

In this section a class of infinite-dimensional systems, called system nodes, are described. In order to give a proper definition of the system node we introduce the following proposition, see (Staffans, $2005, \S 3)$.

Proposition 1. ((Malinen et al., 2003)). Let $X$ be a Hilbert space and let $A: D(A) \subset X \rightarrow X$ be a closed, densely defined linear operator with a nonempty resolvent set $\rho(A)$. Take $\alpha \in \rho(A)$.

(i) Let $X_{1}=D(A)$ and define $\|x\|_{X_{1}}=$ $\|(\alpha-A) x\|_{X}$. Then $\|\cdot\|_{X_{1}}$ is a norm on $X_{1}$ which makes $X_{1}$ into a Hilbert space, and $A \in \mathcal{L}\left(X_{1} ; X\right)$. The operator $(\alpha-A)^{-1}$ maps $X$ isometrically onto $X_{1}$.

(ii) Let $X_{-1}$ be the completion of $X$ with respect to the norm $\|x\|_{X_{-1}}=\left\|(\alpha-A)^{-1} x\right\|_{X}$. Then $X$ is continuously and densely embedded in $X_{-1}$, and $A$ has a unique extension to an operator $A_{e}$ in $\mathcal{L}\left(X ; X_{-1}\right)$. The operator $\left(\alpha-A_{e}\right)^{-1}$ maps $X_{-1}$ isometrically onto $X$. Moreover, $A_{e}$ and $A$ are unitarily similar: $A_{e}=\left(\alpha-A_{e}\right) A\left(\alpha-A_{e}\right)^{-1}$.

(iii) If $A$ is the generator of a $\mathrm{C}_{0}$-semigroup $T(t)$ on $X$, then the restriction $T_{1}(t)=T(t)_{\mid X_{1}}$ of $T(t)$ to $X_{1}$ is a $\mathrm{C}_{0}$-semigroup on $X_{1}$. The semigroup $T(t)$ has a unique extension to a $\mathrm{C}_{0}$-semigroup $T_{e}(t)$ on $X_{-1}$ which is unitarily similar to $T(t)$, since $T_{e}(t)=(\alpha-$ $\left.A_{e}\right) T(t)\left(\alpha-A_{e}\right)^{-1}$.

Note that $X_{1} \subset X \subset X_{-1}$ with continuous and dense embedding. Often $X_{-1}$ is defined in an equivalent way as the dual of $D\left(A^{*}\right)$. Dual version of the spaces $X_{1}$ and $X_{-1}$ can also be constructed by replacing $A$ with the adjoint, $A^{*}$, of $A$. The resulting spaces are denoted by $X_{1}^{d}$ (the equivalent of $X_{1}$ ) and by $X_{-1}^{d}$ (the equivalent of $X_{-1}$ ) respectively. It can be checked that $X_{-1}^{d}$ is the dual of $X_{1}$ with respect to the pivot space $X$. Likewise, $X_{1}^{d}$ is the dual of $X_{-1}$. Thus, $A_{e}^{*} \in$ $\mathcal{L}\left(X ; X_{-1}^{d}\right)$ can be interpreted as the (bounded) adjoint of the operator $A \in \mathcal{L}\left(X_{1} ; X\right)$.

All this is well-know and it is used often in the theory of infinite-dimensional systems.

Now it is possible to define the system node as follows

Definition 2. ((Malinen et al., 2003)). Let $U, X$, and $Y$ be Hilbert spaces. An operator

$$
\mathcal{S}:=\left(\begin{array}{c}
A \& B \\
C \& D
\end{array}\right): D(\mathcal{S}) \rightarrow\left(\begin{array}{c}
X \\
Y
\end{array}\right)
$$

is called an operator node on $(U, X, Y)$ if it has the following structure:

(i) $A$ is a densely defined operator on $X$ with a nonempty resolvent set (which we extend to an operator $A_{e} \in \mathcal{L}\left(X ; X_{-1}\right)$ as explained in Proposition 1);

(ii) $B \in \mathcal{L}\left(U ; X_{-1}\right)$;

(iii) $D(\mathcal{S})=\left\{\left(\begin{array}{l}x \\ u\end{array}\right) \in\left(\begin{array}{l}X \\ U\end{array}\right) \mid A_{e} x+B u \in X\right\}$;

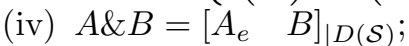

(v) $C \& D \in \mathcal{L}(D(\mathcal{S}) ; Y)$ with respect to the graph norm of $A \& B$ (with values in $X$ ):

$$
\left\|\left(\begin{array}{l}
x \\
u
\end{array}\right)\right\|_{\mathcal{S}}^{2}:=\|x\|_{X}^{2}+\|u\|_{U}^{2}+\left\|A_{e} x+B u\right\|_{X}^{2} .
$$

If in addition to the above, $A$ generates a $\mathrm{C}_{0^{-}}$ semigroup on $X$, then $\mathcal{S}$ is called a system node.

From the boundedness of the operators $A_{e}$ and $B$ combined with the characterization of $D(\mathcal{S})$ it is not difficult to show that the system node $\mathcal{S}$ is a closed operator. It can also be shown that $D(\mathcal{S})$ is dense in $\left[\begin{array}{l}X \\ U\end{array}\right]$.

It is known (see (Malinen et al., 2003)) that if $A$ is the generator of a $\mathrm{C}_{0}$-semigroup then $\mathcal{S}$ defines a linear dynamical system as follows

Proposition 3. ((Malinen et al., 2003)). Let $\mathcal{S}$ be a system node on $(U, X, Y)$. Let $u \in \mathcal{C}^{2}([0, \infty) ; U)$ and $\left[\begin{array}{c}x_{0} \\ u(0)\end{array}\right] \in D(\mathcal{S})$. Then the equation

$$
\left(\begin{array}{c}
\dot{x}(t) \\
y(t)
\end{array}\right)=\mathcal{S}\left(\begin{array}{c}
x(t) \\
u(t)
\end{array}\right), \quad t \geq 0, \quad x(0)=x_{0},
$$

has a unique (classical) solution $\left[\begin{array}{l}x \\ y\end{array}\right]$ satisfying $x \in \mathcal{C}^{1}([0, \infty) ; X) \cap \mathcal{C}^{2}\left([0, \infty) ; X_{-1}\right),\left[\begin{array}{l}x \\ u\end{array}\right] \in$ $\mathcal{C}([0, \infty) ; D(\mathcal{S}))$, and $y \in \mathcal{C}([0, \infty) ; Y)$.

\section{BOUNDARY CONTROL SYSTEMS (BCS)}

As mentioned earlier the class of BCS described here are based on (Curtain and Zwart, 1995, §3.3). That is, BCS of the form 


$$
\begin{aligned}
\dot{x}(t) & =\mathfrak{A} x(t), \quad x(0)=x_{0}, \\
u(t) & =\mathfrak{B} x(t),
\end{aligned}
$$

where $\mathfrak{A}: D(\mathfrak{A}) \subset X \rightarrow X, u(t) \in U$, a separable Hilbert space, and the boundary operator $\mathfrak{B}$ : $D(\mathfrak{B}) \subset X \rightarrow U$ satisfying $D(\mathfrak{A}) \subset D(\mathfrak{B})$, and

Definition 4. The control system (4) is a boundary control system if the following hold:

a. The operator $A: D(A) \rightarrow X$ with $D(A)=$ $D(\mathfrak{A}) \cap \operatorname{ker}(\mathfrak{B})$ and

$$
A x=\mathfrak{A} x \quad \text { for } x \in D(A)
$$

is the generator of a $\mathrm{C}_{0}$-semigroup on $X$.

b. There exists a $B \in \mathcal{L}(U, X)$ such that for all $u \in U, B u \in D(\mathfrak{A})$, the operator $\mathfrak{A} B$ is an element of $\mathcal{L}(U, X)$ and $\mathfrak{B} B u=u$ for $u \in U$.

\section{RELATION OF SYSTEM NODES AND BCS}

In the previous two sections the system node and BCS were discussed. Now it is possible to present some results which relate both representations. To avoid confusion, the control operator of the system node $B$ given in Definition 2 (ii) will be denoted by $B_{\text {node }}$.

Observe that it is not obvious how to find the $B_{\text {node }}$ operator in the case of BCS. For those cases we have the following lemma, which is proved in (Emirsjlow and Townley, 2000).

Lemma 5. In the case of boundary control systems as described in Definition 4, the following relation holds

$$
B_{\text {node }} u=-A_{e} B u+\mathfrak{A} B u,
$$

or equivalently

$$
\begin{aligned}
\langle x| B_{\text {node }} u & >{ }_{D\left(A^{*}\right), D\left(A^{*}\right)^{\prime}} \\
& =-\left\langle A^{*} x, B u\right\rangle+\langle x, \mathfrak{A} B u\rangle .
\end{aligned}
$$

Observe that by using equation (6) it is possible to compute $B_{\text {node }}$ with $A^{*}$ instead of $A_{e}$, which avoids the need of finding the extension, $A_{e}$, of $A$.

Regarding the characterization of $D(\mathcal{S})$ as described in Definition 2 (iii) the following result gives a convenient representation for this domain, which also avoids $A_{e}$.

Lemma 6. For a boundary control system the condition $A_{e} x+B_{\text {node }} u \in X$ on the system node is equivalent to the condition $(x-B u) \in X_{1}$. That is

$$
A_{e} x+B_{\text {node }} u \in X \quad \Longleftrightarrow \quad x-B u \in X_{1}
$$

and hence the domain of $\mathcal{S}$ can be described as

$$
D(\mathcal{S})=\left\{\left(\begin{array}{l}
x \\
u
\end{array}\right) \in\left(\begin{array}{l}
X \\
U
\end{array}\right) \mid x-B u \in X_{1}\right\} .
$$

Furthermore the norm given by (3) is equivalent to the norm

$$
\|x\|_{X}^{2}+\|u\|_{U}^{2}+\|x-B u\|_{X_{1}}^{2} .
$$

PROOF. First condition (7) is proved. Let $A_{e} x+$ $B_{\text {node }} u \in X$. Then we have that

$$
\begin{aligned}
& A_{e} x+B_{\text {node }} u \in X \\
& \Longleftrightarrow\left(\alpha-A_{e}\right)^{-1}\left(A_{e} x+B_{\text {node }} u\right) \in D(A) \\
& \Longleftrightarrow\left(\alpha-A_{e}\right)^{-1} A_{e} x+\left(\alpha-A_{e}\right)^{-1} B_{\text {node }} u \in D(A)
\end{aligned}
$$

and using equation (5) gives

$$
\begin{aligned}
A_{e} x+ & B_{\text {node }} u \in X \\
\Longleftrightarrow(\alpha & \left.-A_{e}\right)^{-1} A_{e} x \\
& +\left(\alpha-A_{e}\right)^{-1}\left(-A_{e} B u+\mathfrak{A} B u\right) \in D(A) \\
\Longleftrightarrow\left(\alpha-A_{e}\right)^{-1} A_{e}(x-B u) & \\
\quad & \left(\alpha-A_{e}\right)^{-1} \mathfrak{A} B u \in D(A) .
\end{aligned}
$$

Recall that $\mathfrak{A} B \in \mathcal{L}(U, X)$ and observe that $\left(\alpha-A_{e}\right)^{-1}$ when restricted to $X$ is equal to the inverse of the resolvent of the semigroup generator, $(\alpha-A)^{-1}$. It thus follows that that $\left(\alpha-A_{e}\right)^{-1} \mathfrak{A} B u(t) \in D(A)$ and hence the condition (8) is equivalent to

$$
\begin{aligned}
A_{e} x+ & B_{\text {node }} u \in X \\
& \Longleftrightarrow\left(\alpha-A_{e}\right)^{-1} A_{e}(x-B u) \in D(A) \\
& \Longleftrightarrow A_{e}(x-B u) \in X \\
& \Longleftrightarrow x-B u \in D(A) .
\end{aligned}
$$

Next the proof of the equivalence of the norms is shown. Let $\left[\begin{array}{l}x \\ u\end{array}\right] \in D(\mathcal{S})$, thus

$$
\begin{aligned}
& \left\|A_{e} x+B_{\text {node }} u\right\|_{X} \\
& =\left\|\left(\alpha-A_{e}\right)\left(\alpha-A_{e}\right)^{-1}\left(A_{e} x+B_{\text {node }} u\right)\right\|_{X} .
\end{aligned}
$$

Since $\left(A_{e} x+B_{\text {node }} u\right) \in X$ one can conclude that $\left(\alpha-A_{e}\right)^{-1}\left(A_{e} x+B_{\text {node }} u\right) \in D(A)=X_{1}$ and since $\left(\alpha-A_{e}\right) D(A)=(\alpha-A) D(A)$ one gets from the equation above that

$$
\begin{aligned}
& \left\|A_{e} x+B_{\text {node }} u\right\|_{X} \\
& =\left\|\left(\alpha-A_{e}\right)^{-1}\left(A_{e} x+B_{\text {node }} u\right)\right\|_{X_{1}} \\
& =\left\|\left(\alpha-A_{e}\right)^{-1}\left(A_{e} x-A_{e} B u+\mathfrak{A} B u\right)\right\|_{X_{1}}(\text { see }(5)) \\
& \leq\left\|\left(\alpha-A_{e}\right)^{-1} A_{e}(x-B u)\right\|_{X_{1}}+\|\mathfrak{A} B u\|_{X} \\
& =\left\|\left(\alpha-A_{e}\right)^{-1}\left(A_{e}-\alpha+\alpha\right)(x-B u)\right\|_{X_{1}}+\|\mathfrak{A} B u\|_{X} \\
& \leq\|x-B u\|_{X_{1}}+|\alpha|\|x-B u\|_{X}+c_{1}\|u\|_{U} \\
& \leq\|x-B u\|_{X_{1}}+|\alpha|\|x\|_{X}+c_{2}\|u\|_{U}
\end{aligned}
$$

Now let $x-B u \in D(A)$ and observe that 


$$
\begin{aligned}
& \|x-B u\|_{X_{1}} \\
& =\|(\alpha-A)(x-B u)\|_{X}=\left\|\left(\alpha-A_{e}\right)(x-B u)\right\|_{X} \\
& =\left\|\alpha x-\alpha B u-A_{e} x+A_{e} B u\right\|_{X} \\
& \leq|\alpha|\|x\|_{X}+c_{3}\|u\|_{U}+\left\|A_{e} x-A_{e} B u\right\|_{X} \\
& =|\alpha|\|x\|_{X}+c_{3}\|u\|_{U} \\
& \quad+\left\|A_{e} x-A_{e} B u+\mathfrak{A} B u-\mathfrak{A} B u\right\|_{X} \\
& \leq|\alpha|\|x\|_{X}+c_{3}\|u\|_{U}+\left\|A_{e} x+B_{\text {node }} u\right\|_{X} \\
& \quad+\|\mathfrak{A} B u\|_{X} \quad(\operatorname{see}(5) \\
& \leq|\alpha|\|x\|_{X}+c_{4}\|u\|_{U}+\left\|A_{e} x+B_{\text {node }} u\right\|_{X} .
\end{aligned}
$$

equations (9) and (10) show that the two norms are equivalent.

\section{BCS RELATED TO SKEW-ADJOINT OPERATOR}

In the paper by (Le Gorrec et al., 2004) the authors associate a $C_{0}$-semigroup with some parameterization of the boundary port variables and define a family of boundary control systems. The form of the main operator studied by them is

$$
\mathcal{J} e=\sum_{i=0}^{N} P(i) \frac{d^{i} e}{d z^{i}}(z) \quad z \in[a, b],
$$

where $e \in C^{\infty}\left((a, b) ; \mathbb{R}^{n}\right)$ and $P(i), i=0, \ldots, N$, is a $n \times n$ real matrix. Since $\mathcal{J}$ is assumed to be skew-symmetric we have

$$
P(i)=P(i)^{T}(-1)^{i+1} .
$$

Here we study the case when $N=1$ and $P(1)$ is assumed to be non-singular. This case includes the well-known wave and beam equations.

Definition 7. The boundary port variables associated with the differential operator $\mathcal{J}$ for $N=1$ are the vectors $e_{\partial}, f_{\partial} \in \mathbb{R}^{n}$, defined by

$$
\left(\begin{array}{l}
f_{\partial} \\
e_{\partial}
\end{array}\right)=R_{\text {ext }}\left(\begin{array}{c}
e(b) \\
e(a)
\end{array}\right)
$$

where $R_{\text {ext }}$ is given by

$$
R_{e x t}=\frac{1}{\sqrt{2}}\left(\begin{array}{cc}
P(1) & -P(1) \\
I & I
\end{array}\right) .
$$

In (Le Gorrec et al., 2004) the authors prove the following theorem.

Theorem 8. Let $W=S(I+V I-V)$, with $S$ invertible and $V V^{T} \leq I$, be a full rank matrix of size $n \times 2 n$, and define $\mathcal{B}: H^{1}\left((a, b), \mathbb{R}^{n}\right) \rightarrow \mathbb{R}^{n}$ as

$$
\mathcal{B} x(t):=W\left(\begin{array}{l}
f_{\partial}(t) \\
e_{\partial}(t)
\end{array}\right) .
$$

Then the system

$$
\begin{aligned}
& \dot{x}(t)=\mathcal{J} x(t), \\
& \mathcal{B} x(t)=u(t)
\end{aligned}
$$

is a boundary control system, where $A_{W}=\mathcal{J}_{\mid \operatorname{ker} \mathcal{B}}$ is the generator of a contraction semigroup with

$$
D\left(A_{W}\right)=\left\{x \in L^{2}\left((a, b), \mathbb{R}^{n}\right) \mid\left(\begin{array}{c}
f_{\partial} \\
e_{\partial}
\end{array}\right) \in \operatorname{ker} W\right\} .
$$

Furthermore, if we define the linear mapping $\mathcal{C}$ : $H^{1}\left((a, b), \mathbb{R}^{n}\right) \rightarrow \mathbb{R}^{n}$ as

$$
\mathcal{C} x(t):=S_{2}\left(I-V^{T}-I-V^{T}\right)\left(\begin{array}{l}
f_{\partial}(t) \\
e_{\partial}(t)
\end{array}\right)
$$

with $S_{2}$ invertible and the output as

$$
y(t)=\mathcal{C} x(t),
$$

then for $u \in C^{2}\left((0, \infty) ; \mathbb{R}^{n}\right)$ and $x(0)-B u(0) \in$ $D\left(A_{W}\right)$ the following balance equation is satisfied:

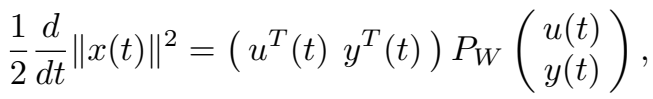

where $P_{W}$ is given by

$\frac{1}{4}\left(\begin{array}{cc}S^{-T}\left(\tilde{P}_{1}^{2}-\tilde{P}_{1} V V^{T} \tilde{P}_{1}\right) S^{-1} & -2 S^{-T} \tilde{P}_{1} V \tilde{P}_{2} S_{2}^{-1} \\ -2 S_{2}^{-T} \tilde{P}_{2} V^{T} \tilde{P}_{1} S^{-1} & S_{2}^{-T}\left(-\tilde{P}_{2}^{2}+\tilde{P}_{2} V^{T} V \tilde{P}_{2}\right) S_{2}^{-1}\end{array}\right)$,

and $\tilde{P}_{1}=\left(I+V V^{T}\right)^{-1}, \tilde{P}_{2}=\left(I+V^{T} V\right)^{-1}$.

Regarding this type of systems we have the following results.

Theorem 9. The boundary control system defined in Theorem 8 is approximately observable.

PROOF. If $u$ and $y$ are set to zero, then all the boundary conditions are equal to zero. In this case, it is not difficult to show that the partial differential equation given by (16) has the unique solution $x(t)=0$. Hence the state $x(t)$ is zero. Consequently, the system is approximatively observable.

It is now clear how to construct the domain of the system node and the operators $A$ and $B_{\text {node }}$ for the BCS of Theorem 8. It can be constructed using the procedure described in Section 4. The only thing left to prove is that the operator $C \& D$ given by the right hand side of (17) is bounded with respect to the norm in (3) (see Definition 2).

Theorem 10. Consider the system (16) with

$$
\mathcal{J} e=P_{0} e(z)+P_{1} \frac{d e}{d z}(z)
$$

and output (17). Then this system can be described by a system node with

$$
\begin{gathered}
A \& B=\left[A_{e} B_{\text {node }}\right]_{\mid D(\mathcal{S})}, \\
D(\mathcal{S})=\left\{\left(\begin{array}{l}
x \\
u
\end{array}\right) \in\left(\begin{array}{c}
X \\
U
\end{array}\right) \mid x-B u \in X_{1}\right\}, \\
C \& D\left(\begin{array}{l}
x(t) \\
u(t)
\end{array}\right)=S_{2}\left(I-V^{T}-I-V^{T}\right)\left(\begin{array}{c}
f_{\partial}(t) \\
e_{\partial}(t)
\end{array}\right),
\end{gathered}
$$

and $B_{\text {node }}$ is given by (5). 
PROOF. We need to check that the five conditions in Def. 2 are satisfied. Condition $(i v)$ is obvious. Condition $(i)$ is a consequence of Theorem 8 , since there it is proved that the system (16) is a BCS (see Def. 4) and since every semigroup generator is closed and densely defined. Condition (ii) follows easily from equation (6). Condition (iii) follows from Lemma 6. Next we prove the boundedness of $C \& D$. First observe that

$$
\begin{aligned}
& C \& D\left(\begin{array}{l}
x \\
u
\end{array}\right)=S_{2}\left(I-V^{T}-I-V^{T}\right)\left(\begin{array}{l}
f_{\partial} \\
e_{\partial}
\end{array}\right) \\
& =S_{2}\left(I-V^{T}-I-V^{T}\right)\left(\begin{array}{cc}
P_{1} & -P_{1} \\
I & I
\end{array}\right)\left(\begin{array}{l}
x(b) \\
x(a)
\end{array}\right) \\
& =C_{1} x(b)+C_{2} x(a)
\end{aligned}
$$

where $C_{1}=S_{2}\left[\left(I-V^{T}\right) P_{1}-\left(I+V^{T}\right)\right]$ and $C_{2}=-S_{2}\left[\left(I-V^{T}\right) P_{1}+\left(I+V^{T}\right)\right]$. It thus follows that for any $\left[\begin{array}{l}x \\ u\end{array}\right] \in D(\mathcal{S})$

$$
\begin{aligned}
& \left\|C \& D\left(\begin{array}{l}
x \\
u
\end{array}\right)\right\|_{\mathbb{R}^{n}}^{2}=\left\|C_{1} x(b)+C_{2} x(a)\right\|_{\mathbb{R}^{n}}^{2} \\
& \leq 2\left\|C_{1} x(b)\right\|_{\mathbb{R}^{n}}^{2}+2\left\|C_{2} x(a)\right\|_{\mathbb{R}^{n}}^{2}
\end{aligned}
$$

and using Lemma 13 gives

$$
\begin{aligned}
\leq & 4(b-a)\left\|C_{1} \frac{d x}{d z}(z)\right\|_{X}^{2}+\frac{4}{(b-a)}\left\|C_{1} x(z)\right\|_{X}^{2} \\
& +4(b-a)\left\|C_{2} \frac{d x}{d z}(z)\right\|_{X}^{2}+\frac{4}{(b-a)}\left\|C_{2} x(z)\right\|_{X}^{2} \\
\leq & k_{1}\left\|\frac{d x}{d z}(z)\right\|_{X}^{2}+k_{2}\|x(z)\|_{X}^{2} \\
\leq & k_{1}\left\|P_{1}^{-1}\right\|^{2}\left\|P_{1} \frac{d x}{d z}(z)+P_{0} x(z)-P_{0} x(z)\right\|_{X}^{2} \\
& +k_{2}\|x(z)\|_{X}^{2} \\
\leq & \tilde{k}_{1}\|\mathcal{J} x(z)\|_{X}^{2}+\tilde{k}_{2}\|x(z)\|_{X}^{2} \\
\leq & 2 \tilde{k}_{1}\|-(\alpha-\mathcal{J})(x(z)-B u)\|_{X}^{2} \\
& +2 \tilde{k}_{1}\|\alpha x(z)-(\alpha-\mathcal{J}) B u\|_{X}^{2}+\tilde{k}_{2}\|x(z)\|_{X}^{2} .
\end{aligned}
$$

By the characterization of $D(\mathcal{S})$ and since $\left[\begin{array}{l}x \\ u\end{array}\right] \in$ $D(\mathcal{S})$ it follows that $x-B u \in D(A)$ and hence $\mathcal{J}=A$. Also recall that $\mathcal{J} B$ is a bounded operator (see Def.4.b). From this we see that

$$
\begin{aligned}
& \left\|C \& D\left(\begin{array}{c}
x \\
u
\end{array}\right)\right\|_{Y}^{2} \leq c_{1}\|(\alpha-A)(x-B u)\|_{X}^{2} \\
& \quad+c_{2}\|x\|_{X}^{2}+c_{3}\|u\|_{X}^{2} \\
& =c_{1}\|x-B u\|_{X_{1}}^{2}+c_{2}\|x\|_{X}^{2}+c_{3}\|u\|_{X}^{2},
\end{aligned}
$$

where $c_{1}, c_{2}, c_{3} \in \mathbb{R}$ are positive constants. Using Lemma 6 we can see that the inequality above implies boundedness of $C \& D$ with respect to the norm in (3).

Theorem 11. Consider the BCS of Theorem 8 with

$$
\mathcal{J} e=P_{1} \frac{d e}{d z}(z) .
$$

(i) If $V=0$, then this system is exponentially stable. (ii) If the matrix $P_{W}$ in (20) is given by

$$
P_{W}=\left(\begin{array}{cc}
I & 0 \\
0 & -I
\end{array}\right),
$$

or equivalently, if the balance equation (19) is given by $\frac{1}{2} \frac{d}{d t}\|x(t)\|^{2}=\|u(t)\|^{2}-\|y(t)\|^{2}$, then the system is exponentially stable and exactly observable in finite time.

PROOF. (i) Observe that if $V=0$ then $W$ has the form $W=S\left(\begin{array}{ll}I & I\end{array}\right)$.

In order to check stability of the system it is necessary to study the resolvent of $A_{W}=\mathcal{J}_{\mid D\left(A_{W}\right)}$. Let $x \in D\left(A_{W}\right)$ and observe that $y(z)=(\lambda-$ $\left.A_{W}\right) x(z)=\lambda x(z)-P_{1} \frac{d x}{d z}(z)$. Thus

$$
\frac{d x}{d z}(z)=\lambda P_{1}^{-1} x(z)-P_{1}^{-1} y(z)
$$

need to be solved. The general solution is given by $x(z)=\mathrm{e}^{\lambda P_{1}^{-1}(z-a)} c-\int_{a}^{z} \mathrm{e}^{\lambda P_{1}^{-1}(z-\tau)} P_{1}^{-1} y(\tau) d \tau$,

where $c$ is a constant vector. Since $x \in D\left(A_{W}\right)$ the boundary conditions (see Theorem 8 ) are described by

$$
\begin{aligned}
& W R_{\text {ext }}\left(\begin{array}{c}
x(b) \\
x(a)
\end{array}\right)=0 \\
\Longleftrightarrow & \left(\begin{array}{ll}
P_{1}+I & I-P_{1}
\end{array}\right)\left(\begin{array}{c}
x(b) \\
x(a)
\end{array}\right)=0 . \quad \text { (see Def. 7) }
\end{aligned}
$$

Using (22) in the equation above yields

$$
\begin{aligned}
\left(P_{1}+I\right) & {\left[\mathrm{e}^{\lambda P_{1}^{-1}(b-a)} c-\int_{a}^{b} \mathrm{e}^{\lambda P_{1}^{-1}(b-\tau)} P_{1}^{-1} y(\tau) d \tau\right] } \\
& +\left(I-P_{1}\right) c=0 \\
\Longleftrightarrow & {\left[\left(P_{1}+I\right) \mathrm{e}^{\lambda P_{1}^{-1}(b-a)}+\left(I-P_{1}\right)\right] c=} \\
& \left(P_{1}+I\right) \int_{a}^{b} \mathrm{e}^{\lambda P_{1}^{-1}(b-\tau)} P_{1}^{-1} y(\tau) d \tau .
\end{aligned}
$$

It follows from Lemma 14 that when $\lambda \in \mathbb{C} \backslash \mathbb{R}^{-}$ the matrix on the left hand side is nonsingular. In that case, $c$ can be defined uniquely, which implies that $\left(\lambda-A_{W}\right)^{-1}$ exists. Also, it is not difficult to show that $\left(\lambda-A_{W}\right)^{-1}$ is bounded, hence (22) defines the resolvent operator of $A_{W}$.

When $\lambda$ is a pure imaginary number, the resolvent (22) is clearly a uniformly bounded operator since $P_{1}$ has only real eigenvalues and hence the magnitude of $\mathrm{e}^{\lambda P_{1}^{-1} z}$ is equal to one. Since $A_{W}$ generates a contraction semigroup it thus follows that the conditions of Theorem 15 are satisfied, which proves the assertion.

(ii) Observe that in this case we also have $V=0$, and hence the system is exponentially stable. Finally, from Theorem 11.3.8 of (Staffans, 2005) one can see that the system is also exactly observable in finite time. 
Remark 12. From the proof of Theorem 11 and Lemma 14 one can see that the eigenvalues (if they exist) of $A_{W}$ are located in $\mathbb{R}^{-}$.

\section{CONCLUSION}

The notion of system node and boundary control systems were studied in this paper. A relation between both was presented. We studied a specific type of systems which are related to a skewsymmetric differentiable operator, for which we showed how to obtain the system node. We also showed that this type of systems is approximately observable. This results also holds for the general differential operator given in (11). Finally, we showed that it is not difficult to obtain an exponentially stable system for a subclass of systems.

\section{APPENDIX}

The following result is not difficult to prove and it is used in the proof of Theorem 10.

Lemma 13. Let $x(z) \in H^{1}\left((a, b) ; \mathbb{R}^{n}\right)$. Then we have that

$$
|x(a)|^{2} \leq 2(b-a)\left\|\frac{d x}{d z}(z)\right\|_{X}^{2}+\frac{2}{(b-a)}\|x(z)\|_{X}^{2},
$$

and

$$
|x(b)|^{2} \leq 2(b-a)\left\|\frac{d x}{d z}(z)\right\|_{X}^{2}+\frac{2}{(b-a)}\|x(z)\|_{X}^{2} .
$$

The next Lemma is used in the proof of Theorem 11.

Lemma 14. If the matrix $P_{1}$ is symmetric and nonsingular then

$$
\left[\left(P_{1}+I\right) \mathrm{e}^{\lambda P_{1}^{-1}(b-a)}+\left(I-P_{1}\right)\right]
$$

is nonsingular if $\lambda \in \mathbb{C} \backslash \mathbb{R}^{-}$. That is, the set of possible $\lambda$ 's that make it singular are located in the negative real axis, $(-\infty, 0)$.

PROOF. First, recall that $P_{1}$ is symmetric and nonsingular. Hence it can be diagonalized, that is $P_{1}=R_{1} D R_{1}^{-1}$ and $P_{1}^{-1}=R_{1} D^{-1} R_{1}^{-1}$, where $R_{1}$ is an orthogonal matrix and $D$ is a diagonal matrix containing the eigenvalues of $P_{1}$ in descending order. Thus that matrix is nonsingular iff the matrix

$R_{1}(D+I) R_{1}^{-1} R_{1} \mathrm{e}^{\lambda D^{-1}(b-a)} R_{1}^{-1}+R_{1}(I-D) R_{1}^{-1}$

is nonsingular. Equivalently, iff

$$
(D+I) \mathrm{e}^{\lambda D^{-1} b}+(I-D)
$$

is nonsingular. This is the same as the diagonal matrix

$$
\left(\begin{array}{cc}
\left(D_{1}+I\right) \mathrm{e}^{\lambda D_{1}^{-1} b}+\left(I-D_{1}\right) & 0 \\
0 & \left(I-D_{2}\right) \mathrm{e}^{-\lambda D_{2}^{-1} b}+\left(I+D_{2}\right)
\end{array}\right)
$$

being nonsingular, where $D_{1}$ is a diagonal matrix containing the positive eigenvalues of $P_{1}$ and $-D_{2}$ contains the negative eigenvalues. Observe that (23) is diagonal and every element of the upper block has the form $(\sigma+1) \mathrm{e}^{\lambda k}+(1-\sigma)$ where $\sigma>0$ and $k>0$ are real numbers. Notice that

$$
\begin{aligned}
& (\sigma+1) \mathrm{e}^{\lambda k}+(1-\sigma)=0 \\
& \Rightarrow \mathrm{e}^{\lambda k}=\frac{\sigma-1}{\sigma+1} \\
& \Rightarrow \mathrm{e}^{\lambda k}<1 \\
& \Rightarrow \lambda \in \mathbb{R}^{-} .
\end{aligned}
$$

Hence the set of possible $\lambda$ 's that make the upper block of (23) singular are located in $(-\infty, 0)$. Using a similar argument it can be proved the same result for the lower block of (23). Altogether proves the lemma.

Theorem 15. (Luo et al., 1999, Corollary 3.36) Let $T(t)$ be a uniformly bounded $C_{0}$-semigroup on a Hilbert space $H$ with generator $A$. Then $T(t)$ is exponentially stable if and only if $i \mathbb{R} \subset \rho(A)$ and

$$
M_{0}:=\sup _{w \in \mathbb{R}}\left\|(i w-A)^{-1}\right\|<\infty .
$$

\section{REFERENCES}

Curtain, R.F. and H.J. Zwart (1995). An Introduction to Infinite-Dimensional Linear Systems Theory. Springer-Verlag. New York.

Emirsjlow, Z. and S. Townley (2000). From PDEs with boundary control to the abstract state equation with unbounded input operator: A tutorial. European J. of Control.

Le Gorrec, Y., H. Zwart and B. Maschke (2004). Dirac structures and boundary control systems associated with skew-symmetric differential operators. Internal report No. 1730, University of Twente (available at http://www.math.utwente.nl/publications/).

Luo, Z.H., B.Z. Guo and O. Morgul (1999). Stability and Stabilization of Infinite Dimensional Systems with Applications. Springer-Verlag.

Malinen, J., O.J. Staffans and G. Weiss (2003). When is a linear system conservative? Report No. 46, 2002/2003, spring, Institute Mittag-Leffler, 36 pp. (avaliable at: http://www.abo.fi/ staffans/publ.htm).

Staffans, O.J. (2005). Well-Posed Linear Systems. Cambridge University Press. 